\title{
RACIOCÍNIO CLÍNICO NA SALA DE URGÊNCIA
}

\author{
CLINICAL REASONING AT THE EMERGENCY DEPARTMENT
}

Fábio F. Neves ${ }^{1}$, Antônio Pazin-Filho²

\begin{abstract}
${ }^{1}$ Médico assistente do Hospital das Clínicas da Faculdade de Medicina de Ribeirão Preto - USP, ${ }^{2}$ Docente do Departamento de Clínica Médica da Faculdade de Medicina de Ribeirão Preto - USP.

Correspondência: Unidade de Emergência do HCRP, Centro de Estudos de Emergência em Saúde, Rua Bernardino de Campos, 1000. Centro, Ribeirão Preto - SP. CEP 14015-130. Telefone: (16) 3602-1225 / Fax: (16) 36021248. (E-mail: fabioneves@ @crp.fmrp.usp.br)
\end{abstract}

Neves FF, Pazin-Filho A. Raciocínio clínico na sala de urgência. Medicina (Ribeirão Preto) 2008; 41 (3): 339-46.

RESUMOS: O raciocínio clínico é o exercício de julgar sobre incerteza durante o cuidado do paciente. Nenhum ambiente médico é tão rico em incertezas quanto à sala de urgência, sendo o raciocínio clínico muitas vezes dificultado por diversos fatores, como a necessidade de tomada de decisões rápidas, sobrecarga de trabalho do médico, equipe mal treinada, dados clínicos incompletos, interrupções freqüentes e pacientes pouco colaborativos. Entretanto, neste ambiente muitas vezes caótico se concentram a maior densidade de decisões na prática médica, muitas delas vitais. Este artigo irá utilizar-se de casos clínicos ilustrativos para descrever primeiramente o modelo clássico de raciocínio clínico, bem como salientar outros modelos que surgem em função das peculiaridades da atuação em emergência, com o objetivo de auxiliar no desenvolvimento de autocrítica para o processo diagnóstico, buscando a redução de erros evitáveis.

Descritores: Raciocínio Clínico. Resolução de Problemas. Tomada de Decisões. Competência Clínica. Medicina de Emergência. Diagnóstico Clínico.

\section{1- RACIOCÍNIO CLÍNICO NA SALA DE UR- GÊNCIA}

O raciocínio clínico pode ser definido como o exercício de julgar sobre incerteza durante o cuidado do paciente ou o caminho cognitivo que o médico segue na tomada de decisão. ${ }^{1 ; 2}$

Nem sempre a prática médica é exercida num contexto baseado em evidências científicas. Muitas vezes, as evidências não estão disponíveis e as decisões são tomadas de forma arbitrária sob a influência da experiência do médico e da expectativa dos pacientes. Para diminuir a possibilidade de erro é fundamental o treinamento dos médicos nos processos cognitivos que auxiliam na tomada de decisão. ${ }^{3}$

$\mathrm{O}$ atendimento às emergências pode apresentar algumas peculiaridades no processo de raciocínio clínico. A sala de urgência é um ambiente rico em incertezas, onde o raciocínio clínico é dificultado por diversos fatores, como a necessidade de tomada de decisões rápidas, sobrecarga de trabalho do médico, equipe mal treinada, dados clínicos incompletos, interrupções freqüentes, além de pacientes e familiares pouco colaborativos. ${ }^{4}$ Entretanto, neste ambiente muitas vezes caótico se concentram a maior densidade de decisões na prática médica, muitas delas vitais. ${ }^{2 ; 5}$

Este artigo irá utilizar-se de casos clínicos ilustrativos para descrever primeiramente o modelo clássico de raciocínio clínico, bem como salientar outros modelos que surgem em função das peculiaridades da atuação na emergência, com o objetivo de auxiliar no desenvolvimento de autocrítica para o processo diagnóstico, buscando a redução de erros evitáveis.

CASO I: o Dr. Prudente chega ao hospital para o seu plantão semanal na Sala de Urgência. 
O primeiro paciente é o Sr. Geraldo, 68 anos, exmilitar. $O$ paciente mostrou-se muito contrariado com a espera e pouco colaborativo.

O Dr. Prudente observa que o Sr. Geraldo está dispnéico, mesmo em repouso, e apesar disto, carrega um maço de cigarros no bolso da camisa. Apesar de utilizar uma vestimenta formal, encontra-se calçando chinelo aberto, não escondendo o edema dos membros inferiores.

A esposa do Sr. Geraldo mostrou-se muito atenciosa, trazendo para a consulta todos os exames realizados e medicamentos prescritos sem sucesso. Dentre as caixas de medicamentos, o médico observou vários tipos diferentes de diuréticos, porém a maioria tinha apenas três ou quatro comprimidos faltando.

Dr. Prudente: - Qual o seu problema de saúde e há quanto tempo ele ocorre?

Sr. Geraldo (com fácies de ansiedade): - Doutor! Eu sinto falta de ar e apresento inchaço pelo corpo há três meses. Já passei por vários médicos e não descobriram o que eu tenho. Os médicos só me mandam parar de fumar e beber. Sempre fui um homem saudável e agora não consigo dormir a noite devido à falta de ar que quase me mata!

Dr. Prudente: - O senhor já está passando mal há três meses?

Sr. Geraldo: - Pensando bem, eu acho que há cerca de 40 dias e piorou esta semana.

Dr. Prudente: - O senhor bebe muito?

Sr. Geraldo: - Não doutor! Eu só bebo socialmente!

Dr. Prudente: - Quantos cigarros o senhor fuma por dia?

Sr. Geraldo: - Depende do dia, mas não passa de uns oito ou dez.

A aquisição de dados é a primeira etapa do raciocínio clínico, fundamental para realização do diagnóstico. Ela deve ser realizada de forma sistemática e cuidadosa, incluindo elementos de história clínica, revisão de prontuário e exame físico. ${ }^{6}$ Para extrair-se o máximo da história clínica devemos utilizar questões abertas e claras, evitando-se perguntas ambíguas ou tendenciosas. $^{7}$

O Dr. Prudente cometeu alguns deslizes na abordagem inicial do caso. Na pergunta " $O$ senhor já está passando mal há três meses?", o paciente pode considerar que o médico está desaprovando a sua procura devido ao quadro arrastado e distorcer para menos o tempo de evolução da doença. Este dado pode ser fundamental na toma de decisões na urgência, como na indicação de terapia trombolítica no infarto agudo do miocárdio.

Já a pergunta "O senhor bebe muito?" é bastante tendenciosa e, provavelmente, o paciente vai responder negativamente. Um questionário aplicado nos Estados Unidos da América sobre a possibilidade de se tornar dependente químico mostrou que $90 \%$ das pessoas se consideram menos suscetíveis que a média da população, contrariando as leis da estatística. ${ }^{8}$ Diante de urgências médicas, mesmo que inconscientemente, os pacientes também tendem a minimizar o número de cigarros fumados. O melhor seria perguntar "Quanto tempo dura um maço de cigarros?" e obter o número indiretamente.

É importante lembrar que as questões sobre hábitos inadequados de vida devem ser formuladas no meio da entrevista, visto que no início o paciente ainda não estabeleceu um vínculo de confiança e no final ele pode querer evitar uma última impressão negativa. Este fenômeno é denominado efeito seqüencial.

Em relação à afirmativa de que a "a falta de ar quase me mata", devemos lembrar-nos do efeito telescópio, onde o paciente tende a superestimar a frequiência de eventos repetidos e a intensidade de eventos agudos. É importante fazer perguntas objetivas que quantifiquem a intensidade e duração do processo, como: no carnaval você já estava sentindo a falta de ar? Você conseguiu subir as rampas deste prédio sem dificuldades?

Após esta breve entrevista, baseado apenas na observação do paciente e na resposta a algumas perguntas abertas, o médico acaba de estabelecer o conceito inicial. ${ }^{9}$ Dados gerais, como aparência, idade, sexo, nível de instrução, postura, expressão facial e linguagem, associados às queixas e aos dados de prontuário (caso existam) vão auxiliar na formação do conceito inicial, o qual não apresenta qualquer organização fisiopatológica ou cronológica relevantes. O conceito inicial é profundamente influenciado pelas condições do atendimento e pelos dados epidemiológicos do paciente.

A próxima etapa do raciocínio clínico é a criação da representação do problema clínico, a qual é uma abstração mental que sintetiza em uma sentença o caso específico. ${ }^{6}$ Muitas vezes os médicos não percebem esta etapa do raciocínio clínico e a realizam intuitivamente. 
Denomina-se problema clínico a queixa que necessita de investigação diagnóstica, pois interfere na qualidade de vida, requerendo uma ação terapêutica ou preventiva por parte do médico. ${ }^{9}$

\section{CASO I - CONTINUAÇÃO}

Baseado neste breve contato, o Dr. Prudente estabelece, rapidamente, o seguinte conceito inicial:

$\begin{array}{lll}\text { Tabagista } & \begin{array}{l}\text { Dispnéia paro- } \\ \text { xística noturna }\end{array} & \begin{array}{l}\text { Idoso } \\ \text { Etilista }\end{array} \\ \text { Ex-militar } & \begin{array}{l}\text { Baixa adesão } \\ \text { terapêutica }\end{array} \\ \text { Dispnéia } & \text { Independente } & \begin{array}{l}\text { Boa estrutura } \\ \text { familiar }\end{array} \\ \text { Edema } & \text { Ansioso } & \begin{array}{l}\text { Sem patologias } \\ \text { prévias }\end{array}\end{array}$

O médico organiza o conceito inicial e gera a seguinte representação do problema: "Homem idoso e tabagista com edema e dispnéia”.

Quase que simultaneamente à formulação da representação do problema clínico, o médico produz as hipóteses diagnósticas. Elas surgem de forma espontânea, em intensidade proporcional à capacidade do médico em conceber explicações plausíveis. A maioria dos médicos formula de três a cinco hipóteses diagnósticas, as quais são produzidas através de heurísticas, que são associações rápidas e práticas entre dados (sinais e sintomas) e explicações potenciais (doença ou síndrome específicas). ${ }^{10}$ Estas heurísticas surgem na memória do médico, intuitivamente, o qual ordena dados oriundos do seu conhecimento (estudo) e de suas vivências clínicas prévias (experiência profissional).

Existem riscos inerentes à utilização das heurísticas, podendo atrasar ou distorcer o diagnóstico clínico. ${ }^{11}$ Uma falha freqüente é a heurística de representação, onde ocorre uma generalização de achados clínicos similares para populações distintas. Por exemplo, quando um paciente apresenta quadro típico de acidente vascular cerebral (AVC), a informação de presença de hálito etílico pode dificultar o diagnóstico, pois o médico já cuidou de vários pacientes embriagados com diminuição do nível de consciência e estes não evoluíram para o diagnóstico de AVC. ${ }^{12} \mathrm{O}$ médico deve ficar atento às correlações ilusórias, onde o efeito é atribuído a uma causa, entretanto sem co- nexão clara do ponto de vista fisiopatológico. A intoxicação etílica não poderia explicar a anisocoria do paciente, mas se o foco estiver em apenas uma hipótese diagnóstica, o profissional tenderia a desprezar este achado.

Outra causa comum de falha diagnóstica é heurística da disponibilidade. Ela ocorre quando o médico, estimulado por fatos marcantes e recentes da prática clínica, tende a procurar com mais ênfase um diagnóstico, valorizando excessivamente os dados que corroboram com a suspeita. Por exemplo, médicos que atenderam recentemente pacientes com bacteremia, tendem a considerar uma probabilidade excessiva deste diagnóstico para os pacientes subseqüentes. ${ }^{13}$ Por outro lado, a ausência de um exame complementar tende a fazer o médico pensar menos nas doenças que exijam este recurso diagnóstico. Na mesma linha de equívoco investigativo está o viés de confirmação, onde o médico direciona sua investigação de forma inflexível para a impressão diagnóstica inicial equivocada. Ele nunca faz questões que possam enfraquecer a suspeita diagnóstica inicial. ${ }^{14}$

Para estabelecer as hipóteses diagnósticas, o médico deve escolher os dados mais relevantes do conceito inicial e se policiar continuamente, evitando vieses. A seleção destes dados deve levar em conta a melhor relação entre sensibilidade e especificidade para o diagnóstico da doença hipotética. Por exemplo, o achado de tufos auriculares é bastante específico para o diagnóstico de gota, assim como a presença de dor articular é bastante sensível. No entanto, a maioria dos pacientes com gota não apresenta tufos auriculares (baixa sensibilidade) e a maioria dos casos de dor articular não são relacionados à gota (baixa especificidade), tendo pouca produtividade a análise isolada destes achados.

Nesta fase, o médico utiliza o denominado conhecimento centrado no dado e busca o maior número possível de doenças que expliquem o problema clínico. Estas doenças são enumeradas de forma hierarquizada, visto que o médico possui um roteiro pré-estabelecido de cada doença em sua base de conhecimentos e priorizará aquelas cujo quadro clínico do paciente mais se correlacione. ${ }^{6}$

\section{CASO I - CONTINUAÇÃO}

O Dr. Prudente seleciona os elementos mais relevantes do conceito inicial e formula a seguinte pergunta: Quais são as principais causas de edema associado à dispnéia que eu conheço? 
1. Insuficiência cardíaca

2. Insuficiência hepática crônica

3. Sindrome nefrítica

4. Síndrome nefrótica

5. Sindrome de Cushing

6. Mixedema

Se ele desejasse poderia fazer, de forma sucessiva, questões mais específicas, como: qual a possibilidade do Sr. Geraldo ter insuficiência cardíaca?

Uma forma consagrada de se testar a hipótese diagnóstica formulada é a utilização do modelo hipotético-dedutivo, descrito por Popper no início do século XX. ${ }^{9}$ Neste modelo, depois de formulado o problema e elaborada a possível solução (hipótese diagnóstica), devemos escolher testes que afastem ou confirmem a doença sugerida. ${ }^{15}$ Um teste pode ser uma pergunta direcionada, uma manobra de exame físico, um exame complementar, ou até mesmo, uma prova terapêutica. Para descartar uma hipótese devemos selecionar testes com alta sensibilidade e para confirmá-la aqueles com alta especificidade. Cada novo resultado de teste auxilia na melhor definição do problema clinico. Agora, o médico utiliza o denominado conhecimento centrado na doença (hipótese diagnóstica) e busca manifestações clínicas e fatores causais típicos da doença hipotética. No caso de uma suspeita de insuficiência cardíaca, por exemplo, o médico deve procurar por condições predisponentes, como tabagismo, diabetes, hipertensão, obesidade, valvulopatia e coronariopatia; sintomas associados como edema, dispnéia aos esforços, ortopnéia e dispnéia paroxística noturna; além de complicações do baixo débito como insuficiência renal e congestão hepática. ${ }^{16}$

CASO I - CONTINUAÇÃO:

Utilizando o modelo hipotético-dedutivo, o Dr. Prudente quer testar a hipótese de insuficiência cardíaca como gênese da dispnéia e do edema do Sr. Geraldo. Logo são formuladas questões que direcionam para fatores de risco e sintomas relacionados a esta patologia:
Dr. Prudente: - Sabe se tem colesterol alto?

Sr. Geraldo: - Estava alto na ultima medida, há dois anos.

Dr. Prudente: - Já teve alguma medida de pressão arterial elevada? É diabético?

Sr. Geraldo: - A pressão sempre está alta. Não tenho diabetes.

Dr. Prudente: - Já apresentou dor no peito, angina ou infarto?

Sr. Geraldo: - Não

Dr. Prudente: - A falta de ar piora a noite ou quando deita?

Sr. Geraldo: - Piora bastante. Eu uso três travesseiros para dormir

Durante o exame físico o Dr. Prudente avalia a presença ou ausência de alterações que possam auxiliar no teste da hipótese de insuficiência cardíaca, como:

- Terceira bulha. Presente.

- Refluxo hepato-jugular. Ausente.

- Ingurgitamento jugular. Presente.

- Estertoração pulmonar. Presente.

- Edema de membros inferiores. Presente.

- Desvio do ictus cardíaco. Ausente.

- Sopro cardíaco. Ausente.

- Arritmia. Presente. Ritmo irregular.

Com estes dados adicionais o Dr. Prudente acredita que a hipótese de insuficiência cardíaca está reforçada e começa a refletir sobre o melhor exame complementar para confirmar o diagnóstico. Ele solicita um eletrocardiograma (ECG) e uma radiografia simples de tórax. No ECG, o médico observa um ritmo de fibrilação atrial, sem outras alterações morfológicas. Na radiografia é evidente a congestão venosa pulmonar, embora a área cardíaca não esteja aumentada.

O médico inicia um tratamento com vasodilatador e diurético de alça e interna o paciente para compensação do quadro de insuficiência cardíaca.

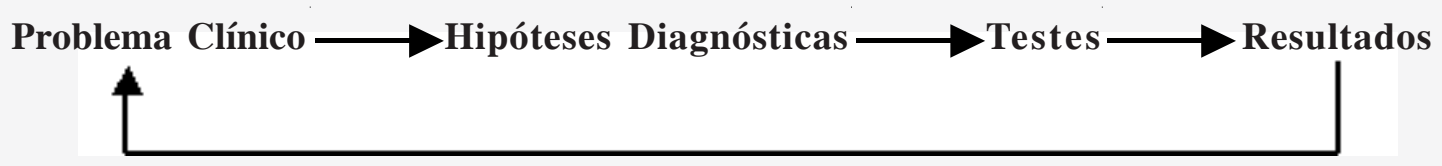

Figura 1: Esquema do modelo hipotético-dedutivo. 
A definição do diagnóstico de insuficiência cardíaca congestiva seguiu uma inferência estatística Bayesina. ${ }^{17} \mathrm{O}$ Teorema de Bayes determina uma abordagem normativa e seqüencial para o processamento da informação.

O conhecimento é processado na forma de hipóteses, caracterizadas por uma probabilidade subjetiva da ocorrência da doença (hipótese verdadeira). A melhor aplicação deste teorema é quando necessitamos rever a hipótese quando uma nova informação é recebida. A nova informação fornece uma razão de verossimilhança que é aplicada na informação antiga, gerando uma probabilidade posterior. ${ }^{18}$

Aplicando o conceito de Bayes no caso clínico acima teríamos a seguinte seqüência estatística: um homem de 68 anos apresenta dispnéia aos esforços. A prevalência de insuficiência cardíaca num homem na sétima década de vida é de aproximadamente $14 \%$ (probabilidade pré-teste). Durante a entrevista clínica, o paciente referiu ter medidas elevadas de pressão arterial. A probabilidade mudou? Segundo o teorema de Bayes sim. A taxa de verossimilhança é determinada pela razão entre a probabilidade de um teste positivo na presença da doença e chance da positividade do teste na ausência de doença (sensibilidade / 1 - especificidade). No caso em questão, a sensibilidade do teste (presença de hipertensão) é $60 \%$ e a especificidade $56 \%$, dando uma razão de verossimilhança de $1,36 .{ }^{19}$ Assim, a probabilidade pós-teste da doença é de $19 \%$ (14\% x 1,36). O paciente também se queixou de dispnéia aos esforços, cuja sensibilidade e especificidade são, respectivamente, $84 \%$ e $34 \%$, determinando uma razão de verossimilhança de 1,27. A nova probabilidade pós-teste é de $24,1 \%$. Durante o exame físico, o médico observou a presença de edema de membros inferiores. Este achado tem uma sensibilidade de $50 \%$ e uma especificidade de $78 \%$. Seguindo a mesma lógica estatística, a nova probabilidade pós-teste para insuficiência cardíaca seria de $54,7 \%$ (24,1\% x 2,27). Após todos os testes positivos, incluindo os achados de exames complementares altamente específicos, como fibrilação atrial no ECG (93\%) e congestão venosa pulmonar na radiografia de tórax (96\%), o médico define com grande margem de segurança o diagnóstico de insuficiência cardíaca.

É importante ressaltar que na prática médica diária, o clínico faz este tipo de análise estatística de forma inconsciente, muitas vezes comparando o caso atual com experiências prévias semelhantes.

Em resumo, o raciocínio clínico clássico compreende a aquisição de informação, a determinação de hipóteses diagnósticas, que são paulatinamente refinadas através de um processo cíclico que envolve o conhecimento do dado e da doença que ampliam a probabilidade de uma determinada hipótese, podendo estar sujeito a diversos erros. A maioria dos casos é conduzida através deste processo cognitivo. Entretanto, as peculiaridades da emergência impõem novos tipos de abordagem que serão descritos a seguir.

CASO II: O segundo paciente é um homem de 50 anos com dor em flanco direito há 20 minutos, em cólica de forte intensidade, que irradiava para a região inguinal, acompanhada de hematúria franca. Apresentava-se pálido, com intensa sudorese e fácies de dor. Imediatamente, o Dr. Prudente faz o diagnóstico de cólica ureteral, solicita que a técnica de enfermagem obtenha acesso venoso periférico e prescreve antiinflamatório endovenoso. Como a melhora da dor é parcial, o médico prescreve analgésico opiáceo, com resolução do sintoma.

O médico acaba de utilizar o método de identificação de padrão. Ele pode ser considerado um "diagnóstico de porta", onde o diagnóstico é freqüentemente óbvio, basicamente visual. ${ }^{15} \mathrm{O}$ problema deste modelo é que o médico pode fechar o diagnóstico prematuramente e equivocadamente (viés de ancoragem).$^{20} \mathrm{O}$ clínico tende a ignorar dados adicionais que refutam o diagnóstico inicial podendo causar dano irreparável. ${ }^{2} \mathrm{O}$ caso II poderia tratar-se de um paciente com dissecção aguda da aorta cujo diagnóstico precoce seria dificultado pela administração de analgésicos e pela decisão de não investigação dos diagnósticos alternativos ao padrão identificado.

CASO III: uma mulher de 65 anos que após lavar o piso da casa apresentou dor torácica de forte intensidade, à direita, sem irradiação, ventilatório-dependente e que piora com a palpação do local. A dor já dura 45 minutos e é caracterizada como muito intensa pela paciente. O exame físico é normal, exceto por hipertensão arterial moderada e discreta taquicardia.

O Dr. Prudente afere a pressão arterial nos quatro membros, solicita radiografia simples de tórax, ECG de 12 derivações, enzimas cardiacas e pesquisa sérica de dímeros $D$. Ele pretende excluir os diagnósticos de tromboembolismo pulmonar, sindrome coronariana aguda, dissecção de aorta e pneumotórax espontâneo, apesar de ter quase certeza de que se trata de uma distensão muscular. 
Neste caso o Dr. Prudente utilizou um método cognitivo denominado exclusão do pior cenário. $\mathrm{O}$ médico faz uma revisão mental das principais doenças de alta mortalidade que devem ser excluídas no quadro clínico. ${ }^{2 ; 21} \mathrm{Se}$ todos estes diagnósticos foram excluídos, provavelmente o paciente não corre risco iminente de morte, podendo o médico avaliar a situação de forma tranqüila e analítica. A principal falha deste método é o fato do médico considerar apenas os diagnósticos mais familiares. Também pode ocorrer retardo no tratamento e utilização ineficaz de recursos visto que a exclusão das doenças potencialmente graves é morosa e cara.

Observe que os métodos de identificação de padrão (CASO II) e exclusão do pior cenário (CASO III) podem ser considerados variações do CASO I. Neles o paciente está consciente, mas apresenta desconforto de tal nível que pode inviabilizar o raciocínio clínico hipotético-dedutivo ou nos quais a utilização de método hipotético-dedutivo pode colocar o paciente em risco inaceitável, frente ao ônus a ser pago na eventualidade de erro diagnóstico. Nestas situações, estes métodos são mais adequados para o atendimento imediato, garantindo a estabilização do paciente até que se tenham condições de prosseguir no processo diagnóstico com segurança.

CASO IV: um paciente de meia idade, sem acompanhante, é trazido inconsciente e direcionado para a sala de emergência. $O$ auxiliar de enfermagem refere que o paciente acaba de perder a consciência. De forma automática o Dr. Prudente executa uma série de ações críticas:

1- Avalia a reatividade do paciente através de estímulos táteis e auditivos: o paciente não reage;

2- Ele solicita que a enfermeira busque o desfibrilador na sala ao lado;

3- Avalia a respiração do paciente: paciente não respira;

4- Pesquisa a possibilidade de corpo estranho obstruindo a via aérea: o tórax expande normalmente após ventilação com bolsa e máscara;

5- Procura a presença de pulso carotídeo: paciente sem pulso;

6- Inicia ressuscitação cárdio-pulmonar com compressões torácicas numa freqüência de 100 por minuto e ventilações com dispositivo bolsa máscara e $100 \%$ de fração inspirada de oxigênio, numa proporção de 30 compressões para duas ventilações;

7- Quando a enfermeira traz o desfibrilador, o Dr. Prudente interrompe as manobras e identifica o ritmo de fibrilação ventricular com as pás do desfibrilador. Instantaneamente, ele aplica um choque elétrico não sincronizado de 360 joules e o paciente volta para ritmo sinusal, com pulso palpável, pressão audivel (70/30 $\mathrm{mmHg})$, porém sem ventilação espontânea.

8- O médico realiza intubação orotraqueal e faz. prova de volume com solução cristalóide, após certificar-se da ausência de congestão pulmonar;

9- Ele prescreve amiodarona (ataque e manutenção), solicita ECG, enzimas cardíacas, radiografia de tórax, eletrólitos e gasometria arterial, transferindo o paciente para o CTI.

Apenas uma hora após estas ações, um familiar é identificado e informa com detalhes os eventos ocorridos com o paciente.

Neste caso, o médico não teve condição de utilizar o modelo hipotético-dedutivo. Ele não conhecia os dados clínicos do paciente, não teve tempo para realizar exame físico completo e não sabia a causa da parada cardíaca. O Dr. Prudente utilizou o método do algoritmo, o qual tenta simplificar o processo de tomada de decisão dividindo-o numa série de estágios. Este método de raciocínio clínico é amplamente divulgado em cursos como o ATLS e o ACLS. ${ }^{22 ; 23}$

Alguns críticos consideram este modelo cognitivo intelectualmente pouco desafiador, mas ele oferece a vantagem de economizar tempo e reduzir a ansiedade do médico, visto que diminui a margem de erro nas situações que necessitam ação rápida, como no atendimento da parada cardíaca. ${ }^{2}$ Entretanto, o uso não crítico deste instrumento pode ser deletério, visto que nem todos os casos podem se enquadrar no padrão esperado. Por exemplo, um algoritmo para tratamento da insuficiência cardíaca descompensada indica a utilização de diurético e nitrato para um paciente dispnéico e com edema de membros inferiores. ${ }^{24}$

Entretanto, o paciente pode ter insuficiência cardíaca diastólica e hipertrofia septal com obstrução da via de saída, sendo o tratamento preconizado no algoritmo potencialmente danoso..$^{25}$

Para o bom uso destes protocolos é fundamental que o médico compreenda adequadamente a base científica da seqüência de decisões determinadas no algoritmo. Assim, ele pode identificar os pacientes que não se adequam às diretrizes.

CASO V: o último paciente da tumultuada manhã do Dr. Prudente é um jovem portador de 
seqüela neurológica grave com alta dependência. A mãe traz o jovem ao hospital porque ele está interagindo menos e deixou de alimentar-se há dois dias. Encontrava-se pálido, diaforético, cianótico e com padrão respiratório superficial. Ao exame foi observada febre aferida em $39^{\circ} \mathrm{C}$, hipotensão, taquicardia e taquipnéia. A ausculta pulmonar evidenciava roncos difusos.

O médico realizou intubação orotraqueal $e$ instalou ventilação mecânica com alta oferta de oxigênio. Foi prescrita reposição volêmica rigorosa e instalado cateter venoso central. Após a administração de fluidos endovenosos sem recuperação dos níveis pressóricos, o médico prescreveu noradrenalina em infusão contínua. O paciente teve a pressão arterial e a oferta tecidual de oxigênio normalizadas. O Dr. Prudente conseguiu leito de terapia intensiva e transferiu o paciente.

O médico utilizou uma estratégia denominada condução do evento. É um método muito utilizado nos departamentos de emergência, que prioriza as ações que preservam a atividade de órgãos nobres, antes mesmo do estabelecimento diagnóstico. ${ }^{2}$ No atendimento médico em questão, o objetivo primordial do Dr. Prudente foi à manutenção das funções vitais, ou seja, o tratamento do choque, mesmo sem ter certeza da etiologia deste. Após a estabilidade clínica do paciente, o médico terá condição de obter dados que possibilitem o diagnóstico etiológico e consequiente tratamento direcionado à causa. É fundamental que a condução do evento seja encarada pelo médico como uma medida ponte e que o diagnóstico etiológico preciso deve ser enfaticamente perseguido.
Os casos IV e V, ao contrário dos casos anteriores, não podem ser considerados variações do CASO I. Neles, os pacientes encontram-se em condições críticas de risco iminente de vida e não há condições de se realizar o raciocínio clínico pelo método hipotético-dedutivo clássico. Para contornar as limitações do processo, estes métodos estão fundamentados em conhecimento prévio e garantem uma atuação praticamente reflexa frente a parâmetros objetivos. Deve-se ter em mente, a exemplo dos casos II e III, que também se tratam de situações paliativas, até que o método clássico possa ser empregado.

\section{2- FIM DO PLANTÃO}

Na sala de urgência temos o ambiente com maior proporção de erros evitáveis, que ocorrem principalmente por falhas no raciocínio clínico. Os erros médicos podem ser causados por falhas intelectuais, más condições ambientais e pela falta de rotina de checagem de erros. ${ }^{1 ; 26}$ A ansiedade presente durante o tratamento de uma emergência leva à redução da capacidade psicomotora e cognitiva dos médicos aumentando a freqüência de erros. ${ }^{4}$

Somado a estas limitações, as situações enfrentadas pelos médicos na sala de urgência impõem novos métodos de raciocínio, que fogem do padrão clássico hipotético-dedutivo e que, apesar de serem necessários e apresentarem vantagens nas situações de avaliação inicial, podem ampliar a margem de erro posterior. O conhecimento destes processos de raciocínio diagnóstico e a limitação decorrente do seu uso podem auxiliar o desenvolvimento de autocrítica e reduzir a ocorrência de erros.

Neves FF, Pazin-Filho A. Clinical reasoning at the emergency department. Medicina (Ribeirão Preto) 2008; 41 (3): 339-46.

ABSTRACT: Clinical reasoning is the exercise of judging with uncertainty in the care of the patient. No medical environment is as unpredictable as to the emergency room, and the clinical reasoning is often hampered by several factors, such as the need for rapid decision-making, work overload, poorly trained staff, incomplete clinical data, frequent interruptions and patient's poor collaboration. However, this environment often chaotic, concentrates the bulk density of decisions on medical practice, many of them vital. This article will use illustrative cases to describe the classic model of clinical reasoning, as well as other models that arise from the peculiarities of the emergency practice, in order to help developing self-assessment in the diagnostic process, seeking the reduction of avoidable errors.

keywords: Clinical Reasoning. Medical Problem Solving. Clinical Decision Making. Clinical Competence. Emergency Medicine. Diagnosis, Clinical. 


\section{REFERÊNCIAS}

1 - Redelmeier DA, Ferris LE, Tu JV, Hux JE, Schull MJ. Problems for clinical judgement: introducing cognitive psychology as one more basic science. CMAJ 2001; 164 (3): 358-60.

2 - Sandhu H, Carpenter C, Freeman K, Nabors SG, Olson A. Clinical decisionmaking: opening the black box of cognitive reasoning. Ann. emerg. med. 2006; 48 (6): 713-19.

3 - Graber ML, Franklin N, Gordon R. Diagnostic error in internal medicine. Arch. intern. med. 2005; 165(13): 1493-99.

4 - Schull MJ, Ferris LE, Tu JV, Hux JE, Redelmeier DA. Problems for clinical judgement: 3 . Thinking clearly in an emergency. CMAJ 2001; 164 (8): 1170-5.

5 - Kovacs G, Croskerry P. Clinical decision making: an emergency medicine perspective. Acad. emerg. med. 1999; 6 (9): 947-52.

6 - Bowen JL. Educational strategies to promote clinical diagnostic reasoning. N. Engl. j. med. 2006; 355(21): 221725.

7 - Redelmeier DA, Schull MJ, Hux JE, Tu JV, Ferris LE. Problems for clinical judgement: 1 . Eliciting an insightful history of present illness. CMAJ 2001; 164 (5): 647-51.

8 - Weinstein ND. Optimistic biases about personal risks. Science 1989; 246 (4935): 1232-3.

9 - Rea-Neto A. Raciocínio clínico - o processo de decisão diagnóstica e terapêutica. Rev Ass Med Brasil 2006; 44(4): 301-11.

10 - Tversky A, Kahneman D. Judgment under Uncertainty: Heuristics and Biases. Science 1974; 185 (4157): 1124-31.

11 - Klein JG. Five pitfalls in decisions about diagnosis and prescribing. BMJ 2005; 330(7494):781-3.

12 - Brannon LA, Carson KL. The representativeness heuristic: influence on nurses' decision making. Appl. nurs. res. 2003; 16(3): 201-4.

13 - Poses RM, Anthony M. Availability, wishful thinking, and physicians' diagnostic judgments for patients with suspected bacteremia. Med. decis. mak. 1991; 11 (3): 159-68.

14 - Wallsten TS. Physician and medical student bias in evaluating diagnostic information. Med. decis. mak. 1981; 1 (2):14564.
15 - Croskerry P. Achieving quality in clinical decision making: cognitive strategies and detection of bias. Acad. emerg. med. 2002; 9 (11): 1184-204.

16 - Jessup M, Brozena S. Heart failure. N. Engl. j. med. 2003; 348 (20): 2007-18.

17 - Gill CJ, Sabin L, Schmid CH. Why clinicians are natural bayesians. BMJ 2005; 330(7499): 1080-3.

18 - Round A. Introduction to clinical reasoning. J. eval. clin. pract. 2001; 7 (2): 109-17.

19 - Wang CS, FitzGerald JM, Schulzer M, Mak E, Ayas NT. Does this dyspneic patient in the emergency department have congestive heart failure? JAMA 2005; 294 (15): 1944-56.

20 - Rottenstreich $\mathrm{Y}$, Tversky A. Unpacking, repacking, and anchoring: advances in support theory. Psychol. rev. 1997; 104 (2): 406-15.

21 - Croskerry P. The cognitive imperative: thinking about how we think. Acad. emerg. med. 2000; 7 (11): 1223-31.

22) Pazin Filho A, Schmidt A, Rosa RM, Castro RBP, Filipini $C$, Rosa MAOF et al. Simulação de pacientes - cursos de suporte de vida ACLS, BLS e PALS na FMRP - USP. Medicina (Ribeirão Preto) 2007; 40 (2): 204-12.

23 - Scarpelini S, Andrade JI. Simulação de pacientes - cursos de suporte de vida. Suporte avançado de vida no trauma. Medicina (Ribeirão Preto) 2007; 40 (2): 199-203.

24 - Silvers SM, Howell JM, Kosowsky JM, Rokos IC, Jagoda AS. Clinical policy: Critical issues in the evaluation and management of adult patients presenting to the emergency department with acute heart failure syndromes. Ann Emerg Med 2007; 49 (5): 627-69.

25 - Nishimura RA, Holmes DR, Jr. Clinical practice. Hypertrophic obstructive cardiomyopathy. N. Engl. j. med. 2004; 350 (13):1320-7.

26 - Redelmeier DA, Koehler DJ, Liberman V, Tversky A. Probability judgement in medicine: discounting unspecified possibilities. Med. decis. mak. 1995; 15 (3): 227-30.

Recebido para publicação em 19/03/2008.

Aprovado para publicação em 14/08/2008. 